\title{
A non-resonant DC magnetic field detector using shear-mode piezoelectric materials
}

\author{
Wei $\mathrm{He}^{1, \mathrm{a}}$, Aichao Yang ${ }^{2, \mathrm{~b}}$, Sixian $\mathrm{Niu}^{1, \mathrm{c}}$ \\ ${ }^{1}$ School of Information Engineering, Baise University, Baise 533000, China \\ ${ }^{2}$ Jiangxi Electric Power Research Institute, Nanchang 330096, China \\ aweiheky@yeah.net, bdkyyac2015@163.com, cjydd0203@126.com
}

Keywords: magnetic field detector, shear-mode, Ampere force, linearity.

Abstract: This paper presents a non-resonant dc magnetic field detector, which is composed of two aluminum strips and shear-mode piezoelectric materials. The operation of the magnetic field detector is based on the Ampere forces resulting from the current-carrying aluminum strips in dc magnetic field and the shear stress on the piezoelectric materials. Experiments have been implemented. The experimental results show that an output voltage of $67.32 \mathrm{mV}$ at $0.22 \mathrm{mT}$ is obtained, and the proposed device has good linearity to the magnetic field.

\section{Introduction}

In recent years, there have been a lot of studies on detecting external magnetic field, including ac and dc magnetic field. The ME laminate composites can sense ac magnetic field with large magnetoelectric coefficients [1-5], and the structures using Lorentz force effect are used to detect dc or quasi-dc magnetic field [6, 7]. The dc magnetic field can operate resonantly or non-resonantly. For the non-resonant devices, the piezoelectric materials typically work in $d_{31}$ mode [6, 7], and the reponse can be further improved.

In this paper, we propose a device for detecting dc magnetic field, which employs the Ampere force and works in $d_{15}$ mode. The $d_{15}$ mode has higher piezoelectric constant and electromechanical coupling factor, which can potentially enhance the response of the device. To verify the viability and effectiveness, a prototype is fabricated and experiments have been carried out. The results show a good linear response to the applied magnetic field, which makes this device potential in real magnetic field sensing.

\section{Magnetic field detector}

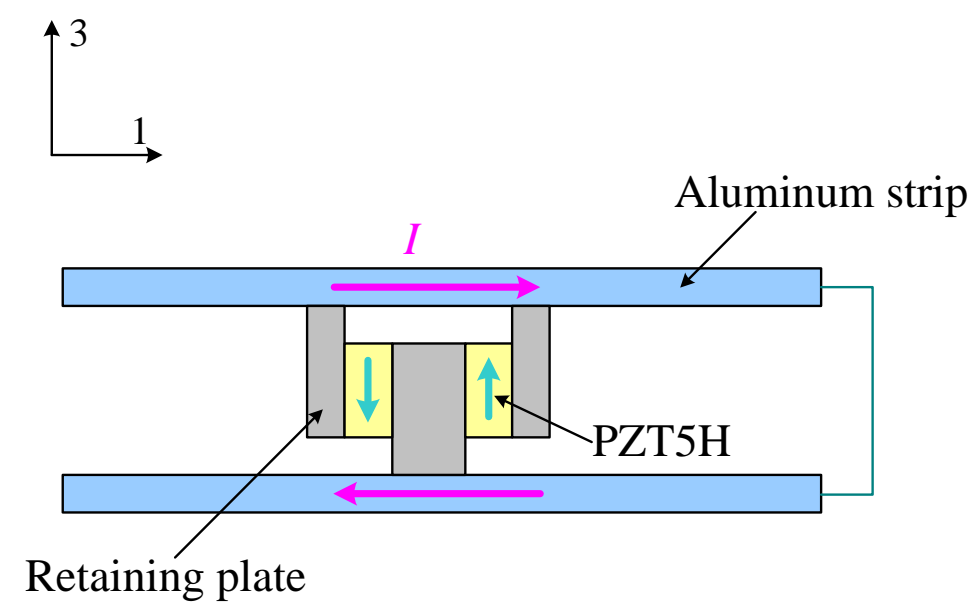

(a) 


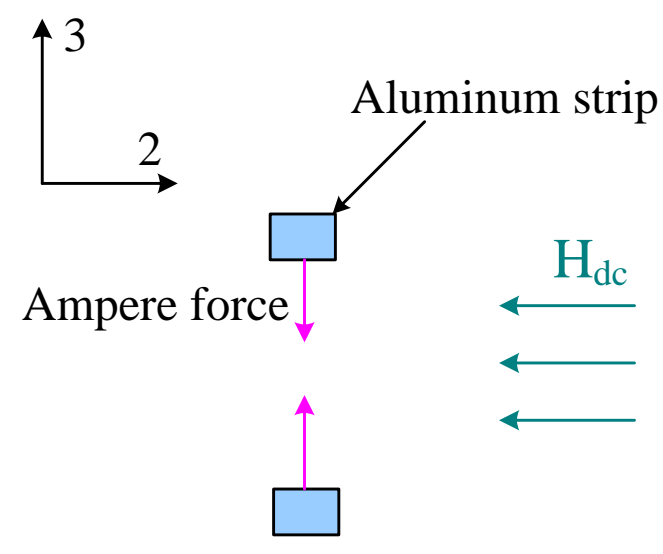

(b)

Fig. 1 Schematic diagram of the dc magnetic field detector

Fig. 1 (a) shows the schematic diagram of the proposed dc magnetic field detector. The detector consists of two piezoelectric plates (shear-mode PZT5H), retaining plates, and two aluminum strips. The two aluminum strips are connected by a wire. The dc magnetic field is along 2-direction, as shown in Fig. 1 (b). When an electric current passes through the aluminum strips, the strips experience Ampere forces with opposite directions, which are plotted in Fig. 1 (b). Assuming that the bottom aluminum strip is fixed, the Ampere force acting on the upper aluminum strip can be expressed as

$$
F_{3}=\mu_{0} H_{d c} I L,
$$

where $\mu_{0}$ is the permeability of vacuum, $H_{\mathrm{dc}}$ is the external dc magnetic field, $I$ is the electric current, and $L$ is the length of the aluminum strip. According to Fig.1, the shear force acting on each PZT5H plate is

$$
F_{s}=\frac{F_{3}}{2}
$$

Then, the shear stress is given by

$$
T_{s}=\frac{F_{3}}{2 l b}=\frac{\mu_{0} H_{d c} I L}{2 l b},
$$

where $l$ and $b$ are the length and the width of the piezoelectric plate, respectively. Under the effect of the retaining plates, the piezoelectric plates work in shear mode and generate voltage output on the electrodes, as shown in Fig. 2. Using the piezoelectric constitutive equations [8], the generated open-circuit voltage can be expressed as

$$
V_{0}=-\frac{d h_{15} T_{s}}{c_{55}^{D}}=-\frac{\mu_{0} d h_{15} H_{d c} I L}{2 l b c_{55}^{D}}
$$

where $h_{15}$ and $c_{55}^{D}$ are respectively the piezoelectric stiffness constant and elastic stiffness coefficient. It can be seen from Eq. 4 that, for the determinate dimensions and material properties are, the output voltage is in direct proportion to the $h_{15}$. 

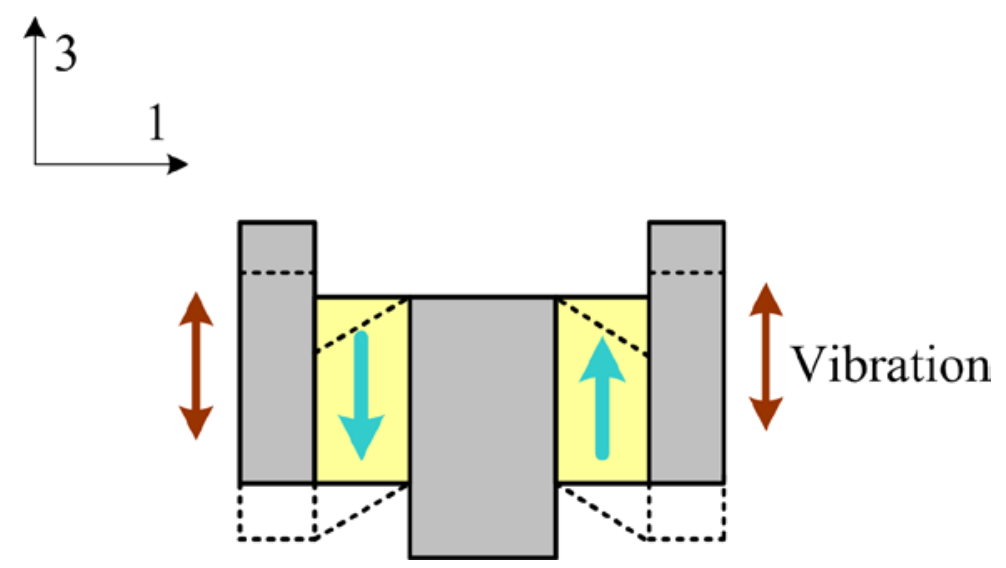

Fig. 2 Shear vibration of the piezoelectric materials

\section{Results and discussions}

Experimental set-up was established and a prototype was fabricated. The two PZT5H plates are connected serially. Fig. 3 shows the induced output voltage versus external dc magnetic field when the electric current in the aluminum strips is $10 \mathrm{~A}$. It can be seen from Fig. 3 that, for a given electric current, the output voltage increases with the applied dc magnetic field (the Ampere forces increase). The output voltage varies from $6.04 \mathrm{mV}$ to $67.32 \mathrm{mV}$ as the dc magnetic field is increased from 0.02 $\mathrm{mT}$ to $0.22 \mathrm{mT}$. In addition, the presented detector has good linearity, which is beneficial in practical magnetic field detecting.

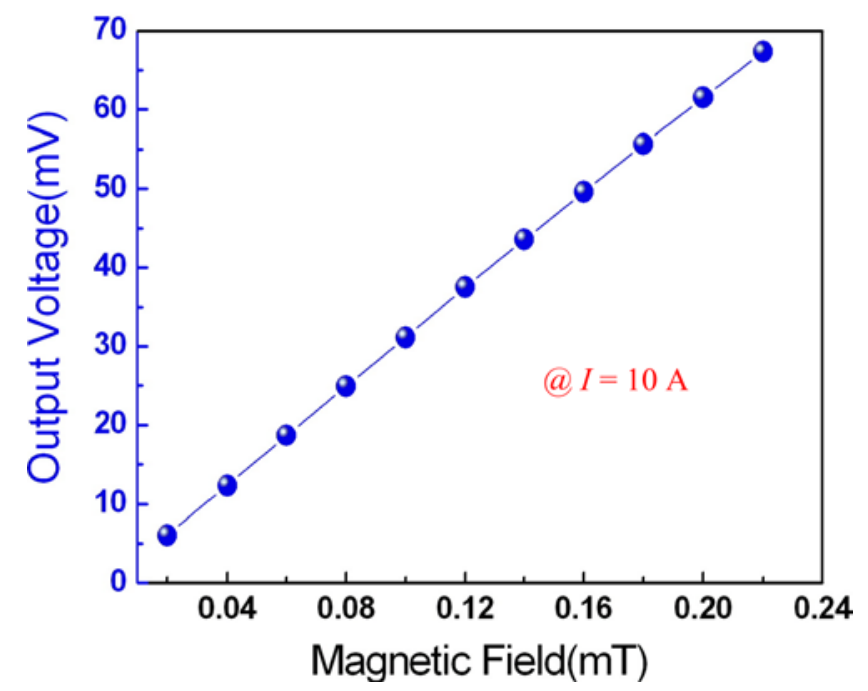

Fig. 3 Output voltage as a function of external dc magnetic field

\section{Conclusions}

In conclusion, we have developed a heterostructure for detecting dc magnetic field. After changing the working mode of the piezoelectric materials compared with the traditional devices, the response of the proposed detector is potentially enhanced. Experimental results show the merits of the detector in practical applications.

\section{Acknowledgments}

This work is supported by the Natural Science Foundation of Guangxi Province (Grant No. 2015GXNSFBA139263). 


\section{References}

[1] Ryu J, Priya S, Uchino K, et al. Magnetoelectric effect in composites of magnetostrictive and piezoelectric materials [J]. Journal of electroceramics, 2002, 8(2): 107-119.

[2] Zhai J, Xing Z, Dong S, et al. Magnetoelectric laminate composites: an overview [J]. Journal of the American Ceramic Society, 2008, 91(2): 351-358.

[3] Lu C, Li P, Wen Y, et al. Enhancement of resonant magnetoelectric effect in magnetostrictive/piezoelectric heterostructure by end bonding [J]. Applied Physics Letters, 2013, 102(13): 132410.

[4] Filippov D A, Laletsin U, Srinivasan G. Resonance magnetoelectric effects in magnetostrictive-piezoelectric three-layer structures [J]. Journal of Applied Physics, 2007, 102(9): 093901.

[5] Chen L, Li P, Wen Y, et al. Resonance magnetoelectric couplings of piezoelectric ceramic and ferromagnetic constant-elasticity alloy composites with different layer structures [J]. Journal of Alloys and Compounds, 2013, 555: 156-160.

[6] Jia Y M, Zhou D, Luo L H, et al. Magnetoelectric effect from the direct coupling of the Lorentz force from a brass ring with transverse piezoelectricity in a lead zirconate titanate (PZT) disk [J]. Applied Physics A: Materials Science \& Processing, 2007, 89(4): 1025-1027.

[7] Leung C M, Or S W, Ho S L. dc magnetoelectric sensor based on direct coupling of Lorentz force effect in aluminum strip with transverse piezoelectric effect in $0.7 \mathrm{~Pb}\left(\mathrm{Mg}_{1 / 3} \mathrm{Nb}_{2 / 3}\right) \mathrm{O}_{3}-0.3 \mathrm{PbTiO}_{3}$ single-crystal plate [J]. Journal of Applied Physics, 2010, 107(9): 09E702.

[8] Ren B, Or S W, Zhang Y, et al. Piezoelectric energy harvesting using shear mode $0.71 \mathrm{~Pb}(\mathrm{Mg}$ 1/3 $\left.\mathrm{Nb}_{2 / 3}\right) \mathrm{O}_{3}-0.29 \mathrm{PbTiO}_{3}$ single crystal cantilever [J]. Applied Physics Letters, 2010, 96(8): 083502. 